Supporting Information for:

\title{
Hydrogen Evolution Reaction Monitored by Electrochemiluminescence Blinking at Single-Nanoparticle Level
}

Cheng Ma ${ }^{\mathrm{a}}$, Hui-Fang Weia, Min-Xuan Wang ${ }^{\mathrm{a}}$, Shaojun $\mathrm{Wu}^{\mathrm{a}}$, Yu-Chung Chang ${ }^{\mathrm{b}}$, Jianrong Zhang ${ }^{\mathrm{a}}$, Li-Ping Jiang ${ }^{\mathrm{a}}$, Wenlei Zhu ${ }^{\mathrm{b}, *}$, Zixuan Chen ${ }^{\mathrm{a}, *}$, and Yuehe Lin ${ }^{\mathrm{b}, *}$

aState Key Laboratory of Analytical Chemistry for Life Science School of Chemistry and Chemical Engineering, Nanjing University, Nanjing 210023, P. R. China.

${ }^{b}$ School of Mechanical and Materials Engineering, Washington State University, Pullman, WA 99164, USA.

\section{Contents}

1. Characterization of hollow carbon nitride nanosphere (HCNS) ………………................S2

2. ECL microscopy for single-nanoparticle analysis.............................................................. 3

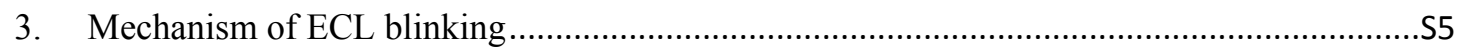

4. Characterization of AuPd/HCNS, NiS/HCNS and Pt/HCNS............................................... 10

5. ECL blinking causes the low cathodic ECL efficiency....................................................

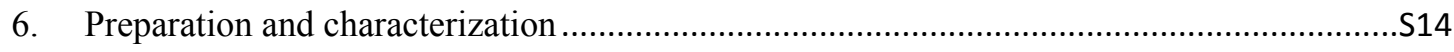

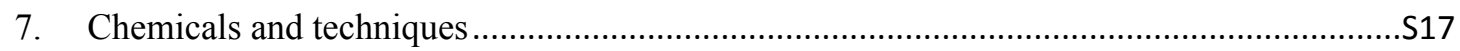

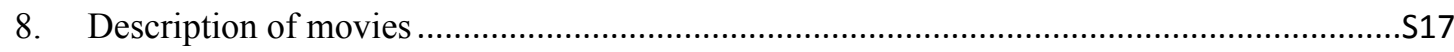

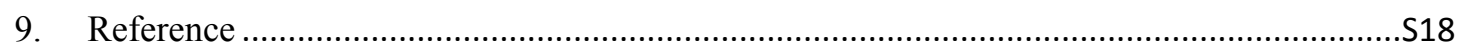


1. Characterization of hollow carbon nitride nanosphere (HCNS)
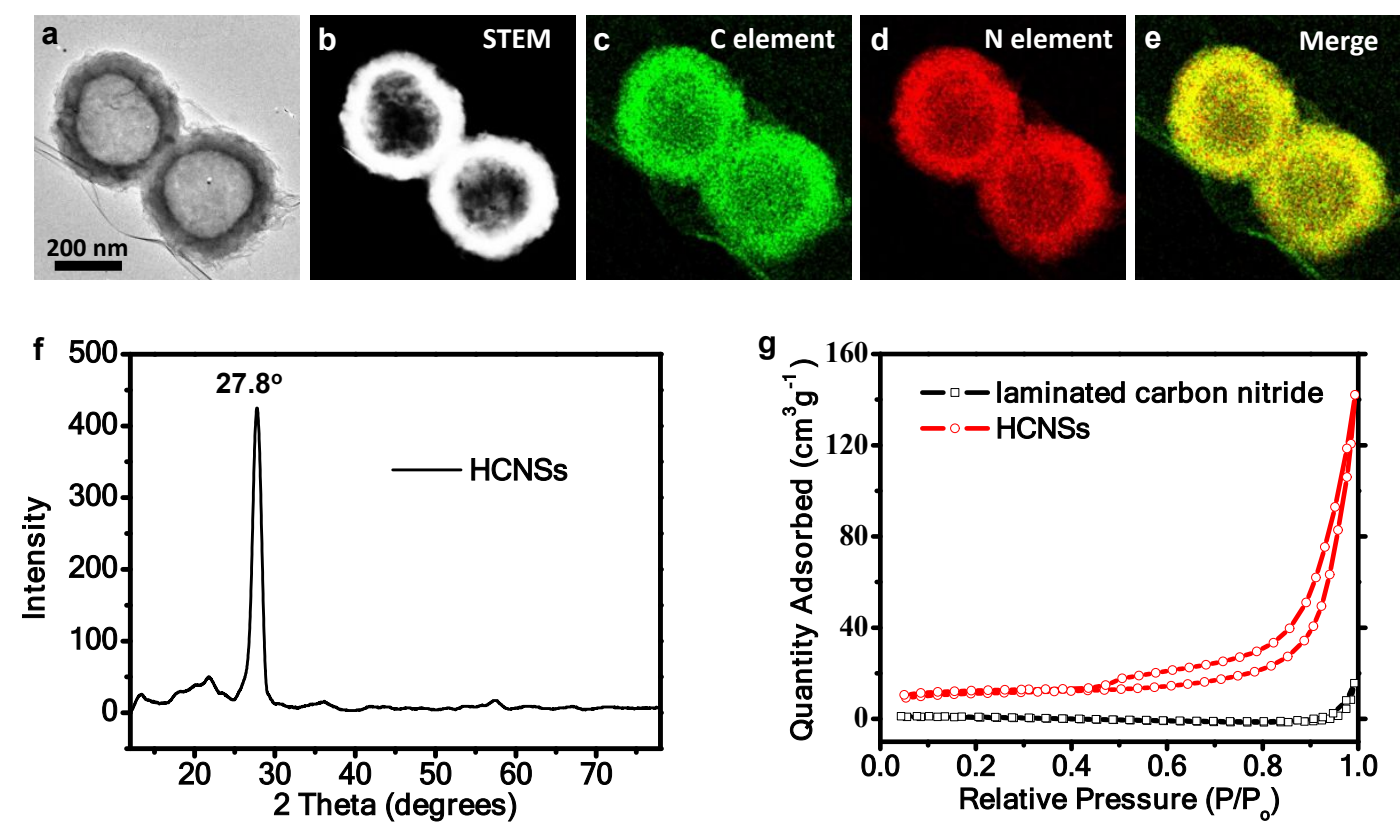

Figure S1. Transmission electron microscopy (TEM) image (a), EDX high-angle annular dark field (HAADF) STEM image (b) and STEM-EDX elemental maps (c-e) of HCNS. (f) XRD patterns of HCNS. The XRD peak at $27.8^{\circ}$ is attributed to the (002) reflection of a graphitic-like aromatic structure. (g) Nitrogen adsorption-desorption isotherms of laminated carbon nitride and HCNS. BET specific surface values calculated from the nitrogen adsorption-desorption isotherms are $1.217 \mathrm{~m}^{2} / \mathrm{g}$ and $36.036 \mathrm{~m}^{2} / \mathrm{g}$ for laminated carbon nitride and HCNS, respectively.

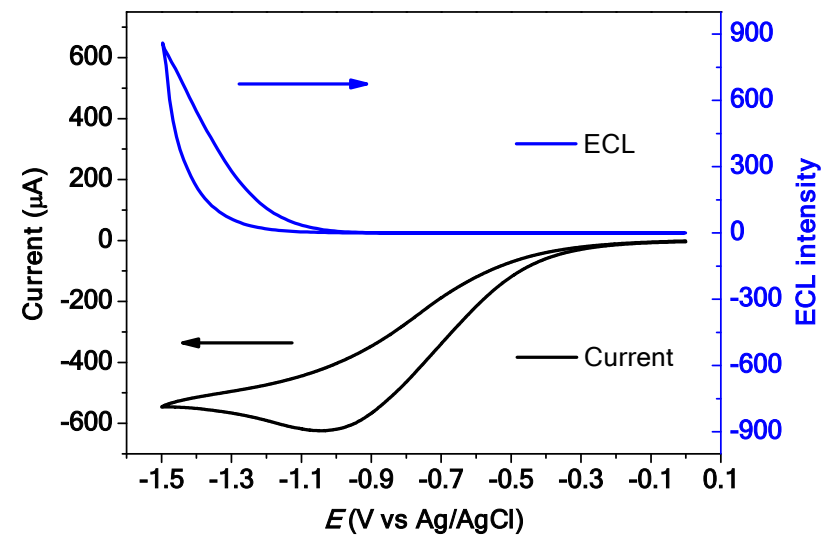

Figure S2. ECL-potential curve and cyclic voltammetry curve of $\mathrm{HCNS}$ with $100 \mathrm{mM} \mathrm{K}_{2} \mathrm{SO}_{4}$ as the coreactant in100 $\mathrm{mM} \mathrm{K}_{2} \mathrm{SO}_{4}$ electrolyte. Scan rate: $0.1 \mathrm{~V} / \mathrm{s}$. 


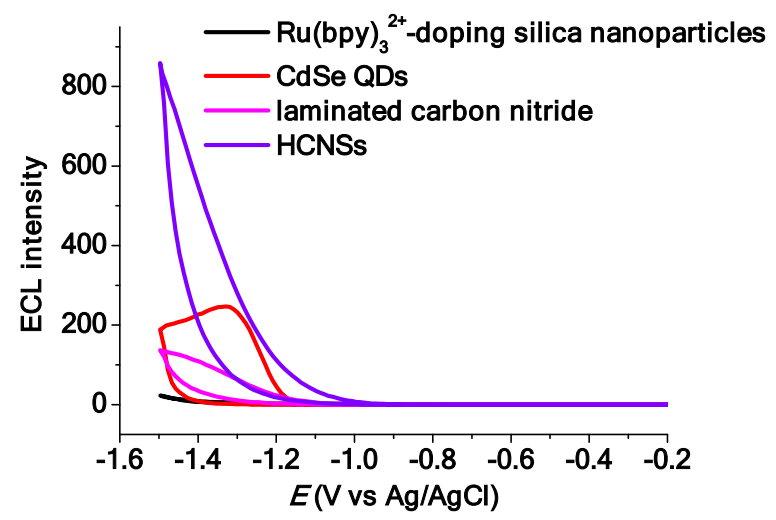

Figure S3. ECL-potential curves of $\mathrm{Ru}(\mathrm{bpy})_{3}{ }^{2+}$-doping silica nanoparticle, CdSe quantum dot, laminated carbon nitride, and $\mathrm{HCNS}$ in the electrolyte of $100 \mathrm{mM} \mathrm{K}_{2} \mathrm{SO}_{4}$ containing $100 \mathrm{mM} \mathrm{K} \mathrm{S}_{2} \mathrm{O}_{8}$ coreactant with a cyclic voltammetry scan between $0.0 \mathrm{~V}$ and $-1.5 \mathrm{~V}$. The quantities of all these four materials on GC electrodes are $5 \mu \mathrm{g}$.

\section{ECL microscopy for single-nanoparticle analysis}

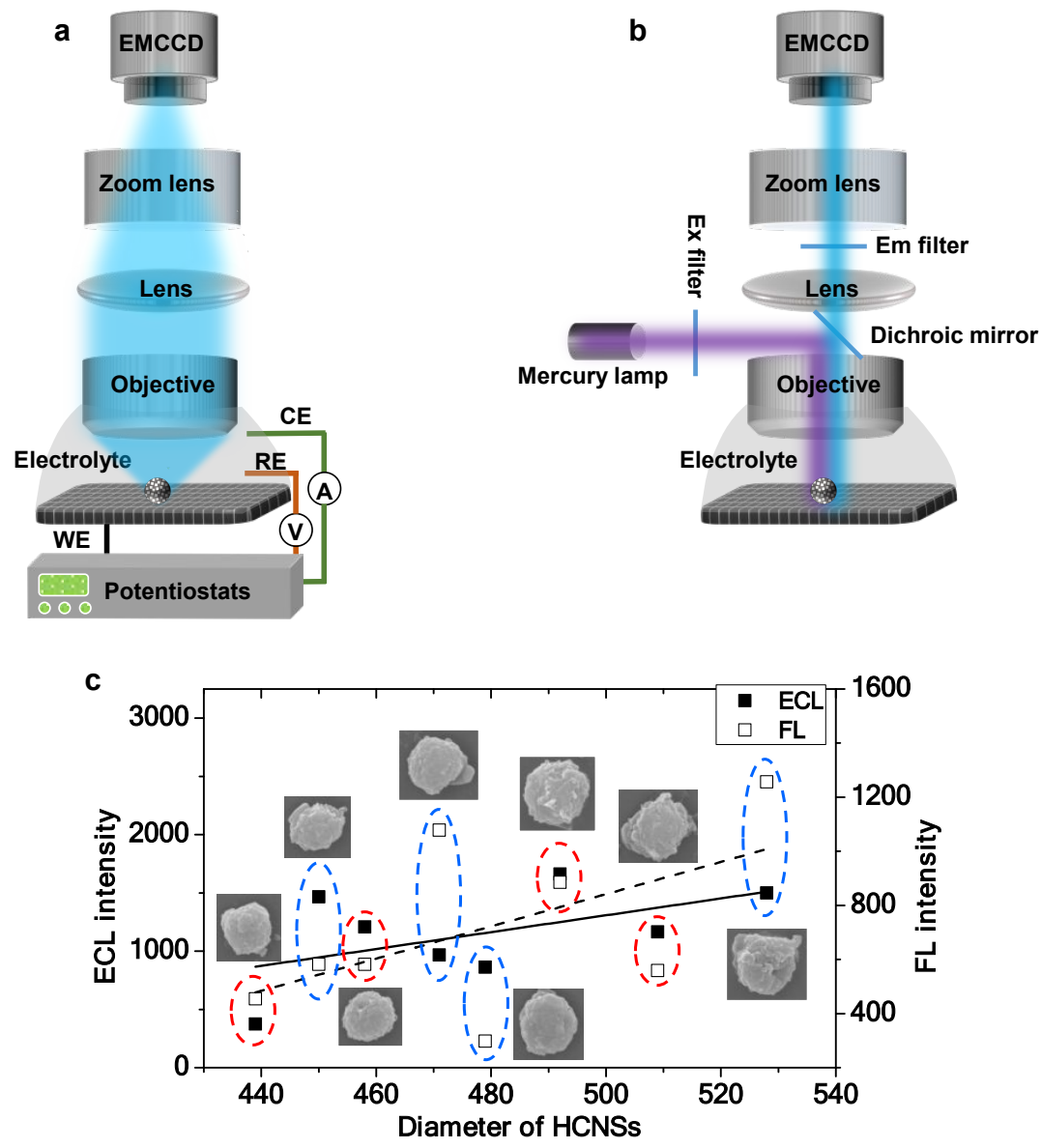

Figure S4. (a) Schematic illustration of the ECL microscopy setup. Application of a voltage to the work electrode results in the ECL emission of HCNS, which is collected by a water-immersion objective of high numerical aperture. The diverging light is focused by the imaging lens and then is imaged on the EMCCD. (b) Schematic illustration of 
the correlated fluorescence (FL) microscopy setup based on the homemade ECL microscopy with a white light source (mercury lamp), a dichroic mirror (Reflection band 360/407 nm; transmission band 425/575 nm), and filters (Excitation bandpass filter $381 / 399 \mathrm{~nm}$; emission bandpass filter 430/490 nm). The high-magnification zoom lens systems $(0.7-4.5 \mathrm{X}$ magnification range) in front of EMCCD camera offer variable imaging vision. ECL and FL microscopy use the same patterned glassy carbon electrode for further SEM analysis. (c) ECL and FL variations with different sizes of individual HCNSs. Insets, SEM images of HCNS corresponding their ECL and FL intensity. Solid and dash lines represent the linear fitting curves of ECL intensity and FL intensity, respectively. The red and blue circles denote the strong and weak correlations between ECL and FL intensity, respectively.
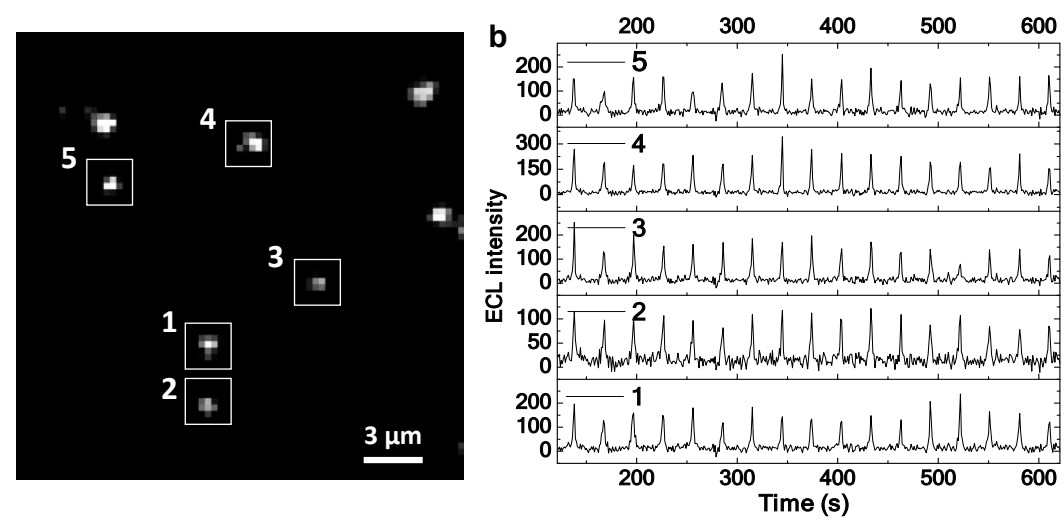

Figure S5. Stability of the ECL intensities of five HCNSs when the potential is cyclically scanned from $0 \mathrm{~V}$ to -1.5 $\mathrm{V}$ at a scan rate of $100 \mathrm{mV} / \mathrm{s}$ in $100 \mathrm{mM} \mathrm{K}_{2} \mathrm{SO}_{4}$ containing $100 \mathrm{mM} \mathrm{K}_{2} \mathrm{~S}_{2} \mathrm{O}_{8}$ coreactant. (a) The ECL image of discrete HCNSs on the glassy carbon electrode. (b) The ECL-time curves of five HCNSs marked 1-5 in (a) with successive CV scans. 


\section{Mechanism of ECL blinking}
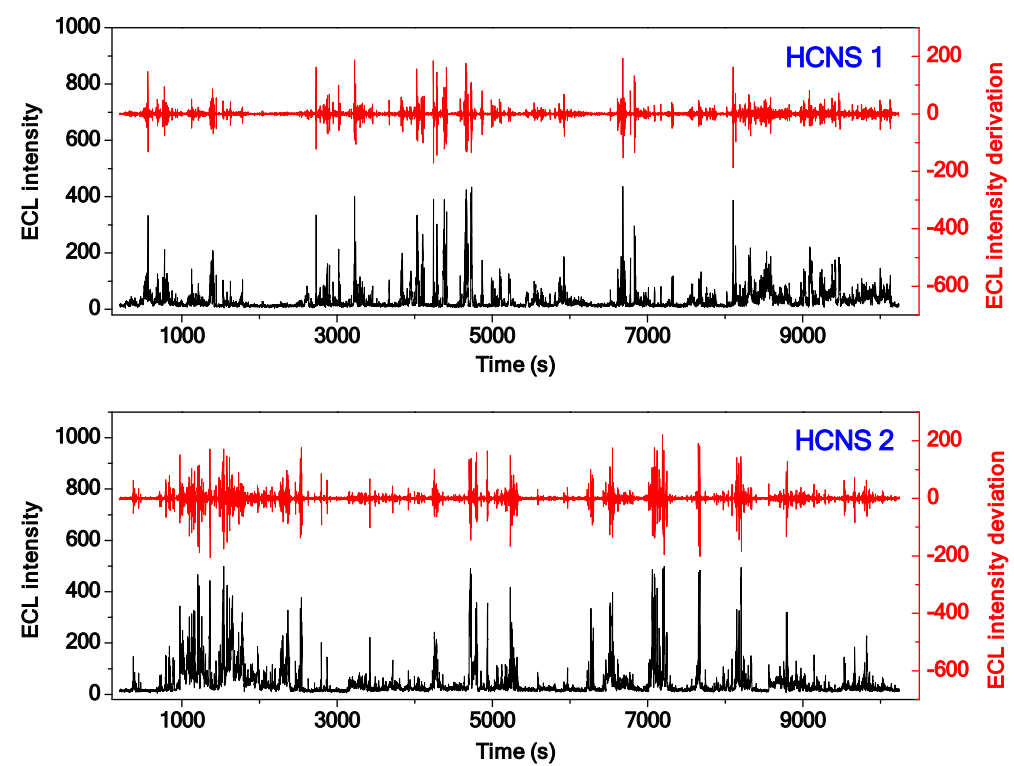

Figure S6. ECL trajectories of two single HCNSs (black lines) and correlation analyses (red lines) of the two ECL trajectories to investigate the intensity variation with time elapsing.

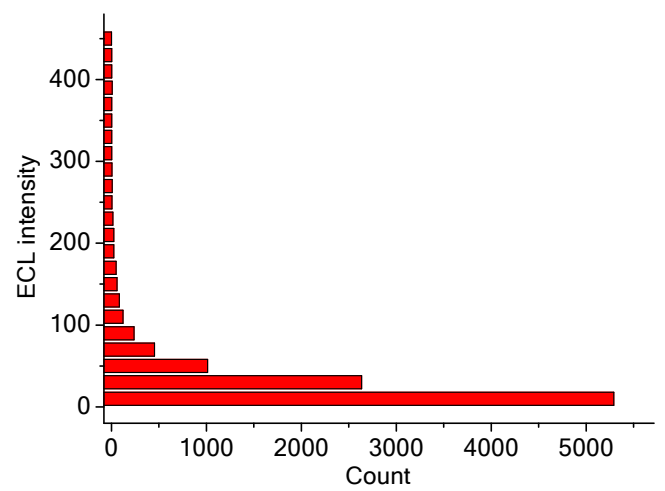

Figure S7. Histogram of frequency distribution of the corresponding ECL intensities. The Y-axis is a linear scale of ECL intensities.
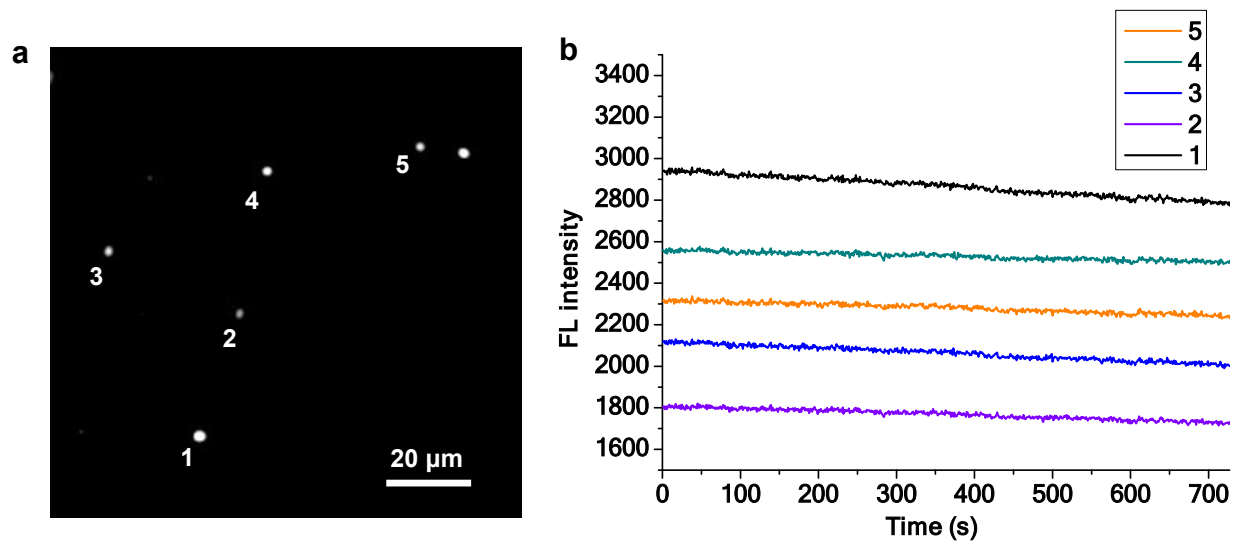

Figure S8. (a) FL image of HCNSs. (b) The FL trajectories of five HCNSs marked in (a). The exposure time is $1 \mathrm{~s}$. 


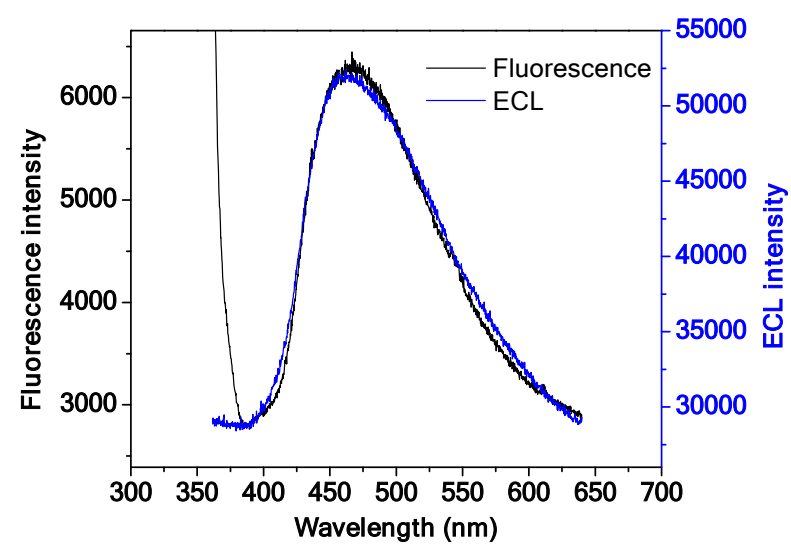

Figure S9. ECL and FL spectra of HCNS. Excitation wavelength of fluorescence: $350 \mathrm{~nm}$. ECL spectrum is obtained at constant voltage $-1.5 \mathrm{~V}$ with $100 \mathrm{mM} \mathrm{K}_{2} \mathrm{~S}_{2} \mathrm{O}_{8}$ as the coreactant.
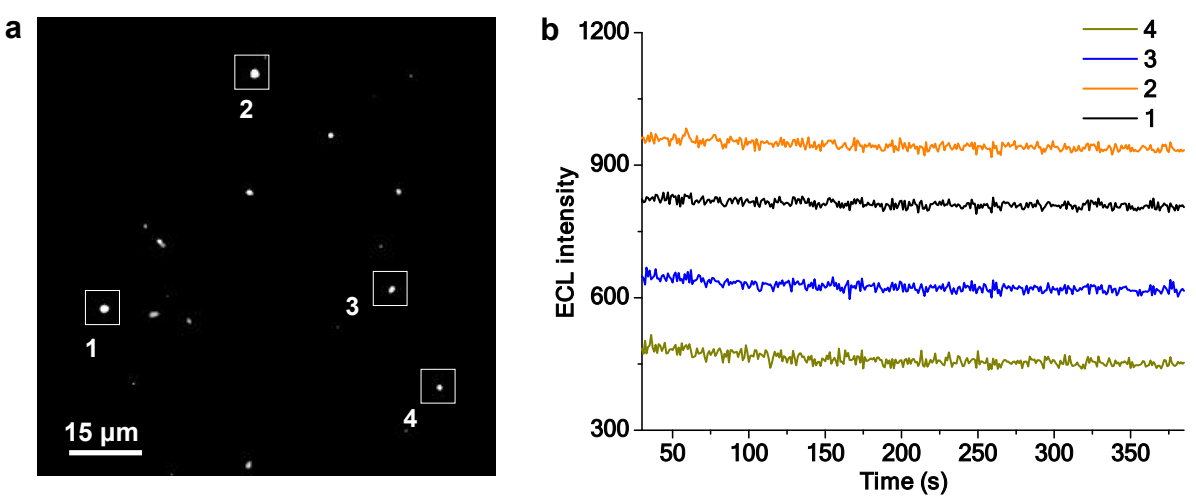

Figure S10. (a) Anodic ECL image of HCNSs. (b) The ECL trajectories of four HCNSs marked in (a). The potential is constant $1.6 \mathrm{~V}$ in $200 \mathrm{mM}$ PBS buffer $(\mathrm{pH}=7.0)$ containing $100 \mathrm{mM}$ tri-n-propylamine (TPrA). The exposure time is $1 \mathrm{~s}$. The ECL image is an accumulation of 30 bright original ECL images to improve the signal to noise ratio.

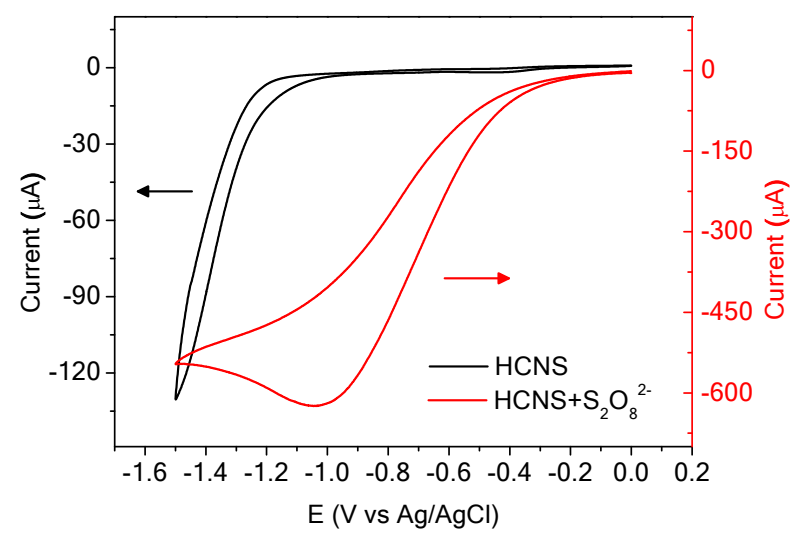

Figure S11. CV curves of HCNSs on GC electrode in either $100 \mathrm{mM} \mathrm{K}_{2} \mathrm{SO}_{4}$ (black line) or $100 \mathrm{mM} \mathrm{K}_{2} \mathrm{SO}_{4}$ containing $100 \mathrm{mM} \mathrm{K} \mathrm{S}_{2} \mathrm{O}_{8}$ coreactant (red line). The rising current beyond $-1.1 \mathrm{~V}$ in black line suggests electrocatalytic HER on HCNSs. The rising current in red line suggests both electrochemical reduction of $\mathrm{K}_{2} \mathrm{~S}_{2} \mathrm{O}_{8}$ and electrocatalytic HER on HCNSs. At $-1.5 \mathrm{~V}$, the reduction current of HCNS in the presence of $100 \mathrm{mM} \mathrm{S}_{2} \mathrm{O}_{8}{ }^{2-}$ rises fourfold than that without $100 \mathrm{mM} \mathrm{S}_{2} \mathrm{O}_{8}{ }^{2-}$. 
a
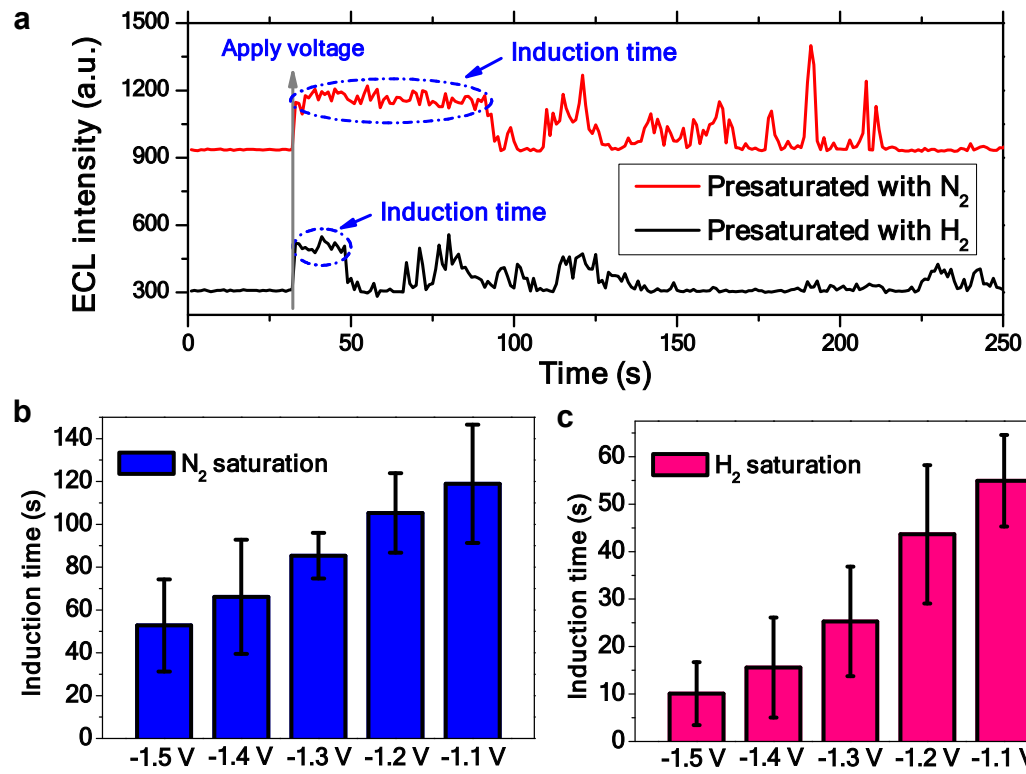

C

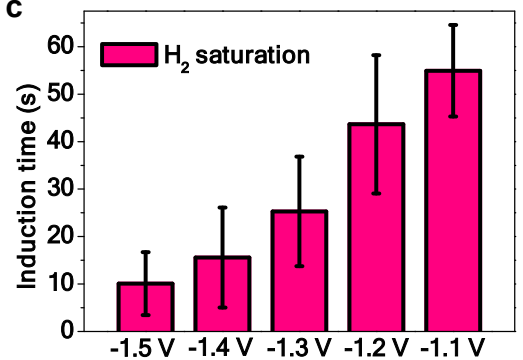

Figure S12. Induction time of ECL blinking in different electrolytes and at different electrode potentials. (a) ECL trajectories of single HCNS at constant $-1.5 \mathrm{~V}$ potential in the electrolyte presaturated with nitrogen gas and hydrogen gas, respectively. The electrolyte is $100 \mathrm{mM} \mathrm{K}_{2} \mathrm{SO}_{4}$ containing $100 \mathrm{mM} \mathrm{K}_{2} \mathrm{~S}_{2} \mathrm{O}_{8}$ coreactant. Statistics of induction time of single HCNS at different electrode potentials in $\mathrm{N}_{2}$ saturated (b) and $\mathrm{H}_{2}$ saturated (c) electrolytes, respectively.

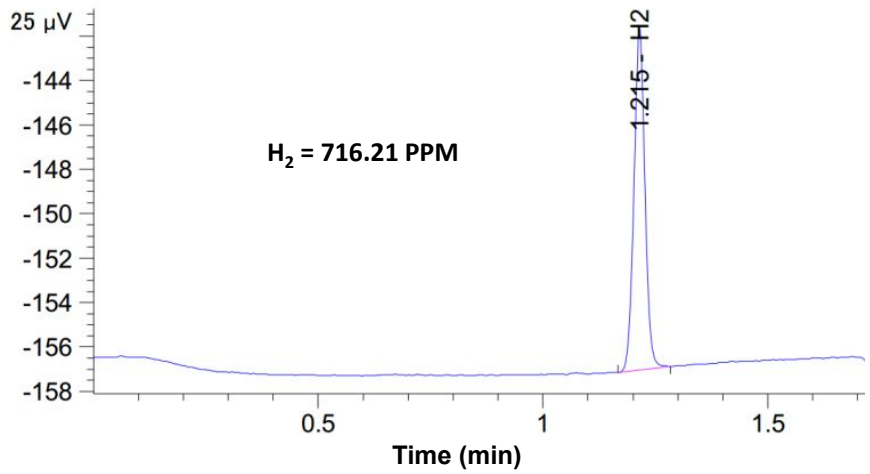

Figure S13. Gas chromatogram of the gaseous products during ECL blinking at the constant $-1.5 \mathrm{~V}$ potential in 100 $\mathrm{mM} \mathrm{K}_{2} \mathrm{SO}_{4}$ containing $100 \mathrm{mM} \mathrm{K}_{2} \mathrm{~S}_{2} \mathrm{O}_{8}$ coreactant. 


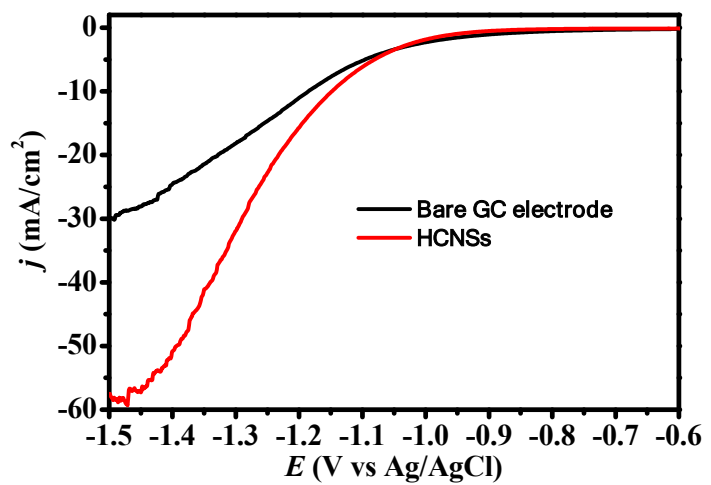

Figure S14. Polarization curves for hydrogen evolution reaction (HER) of bare GC electrode and HCNSs modified $\mathrm{GC}$ electrode in $500 \mathrm{mM} \mathrm{H}_{2} \mathrm{SO}_{4}$ electrolyte at a scan rate of $5 \mathrm{mV} / \mathrm{s}$.
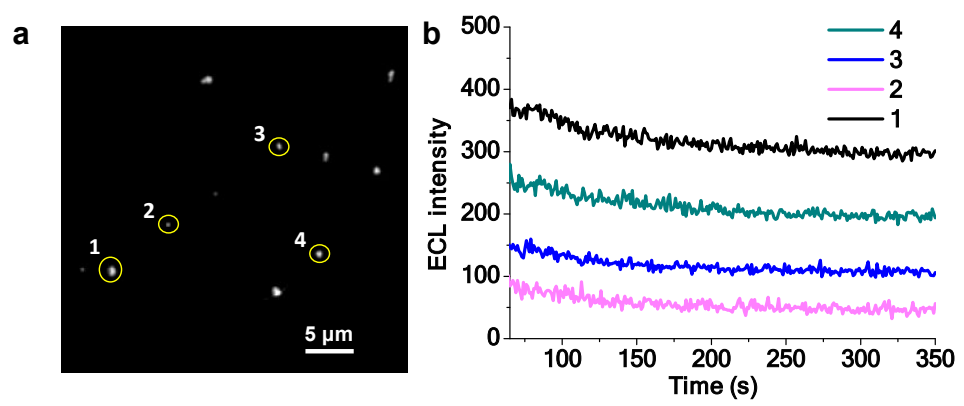

Figure S15. (a) Cathodic ECL image of RuDSN (500 nm). (b) ECL trajectories of four RuDSNs marked in (a) when a constant voltage (-1.5 V) is imposed on the GC electrode in $100 \mathrm{mM} \mathrm{K}_{2} \mathrm{SO}_{4}$ containing $100 \mathrm{mM} \mathrm{K}_{2} \mathrm{~S}_{2} \mathrm{O}_{8}$ coreactant. The ECL image is an accumulation of 50 bright original ECL images to improve the signal to noise ratio.
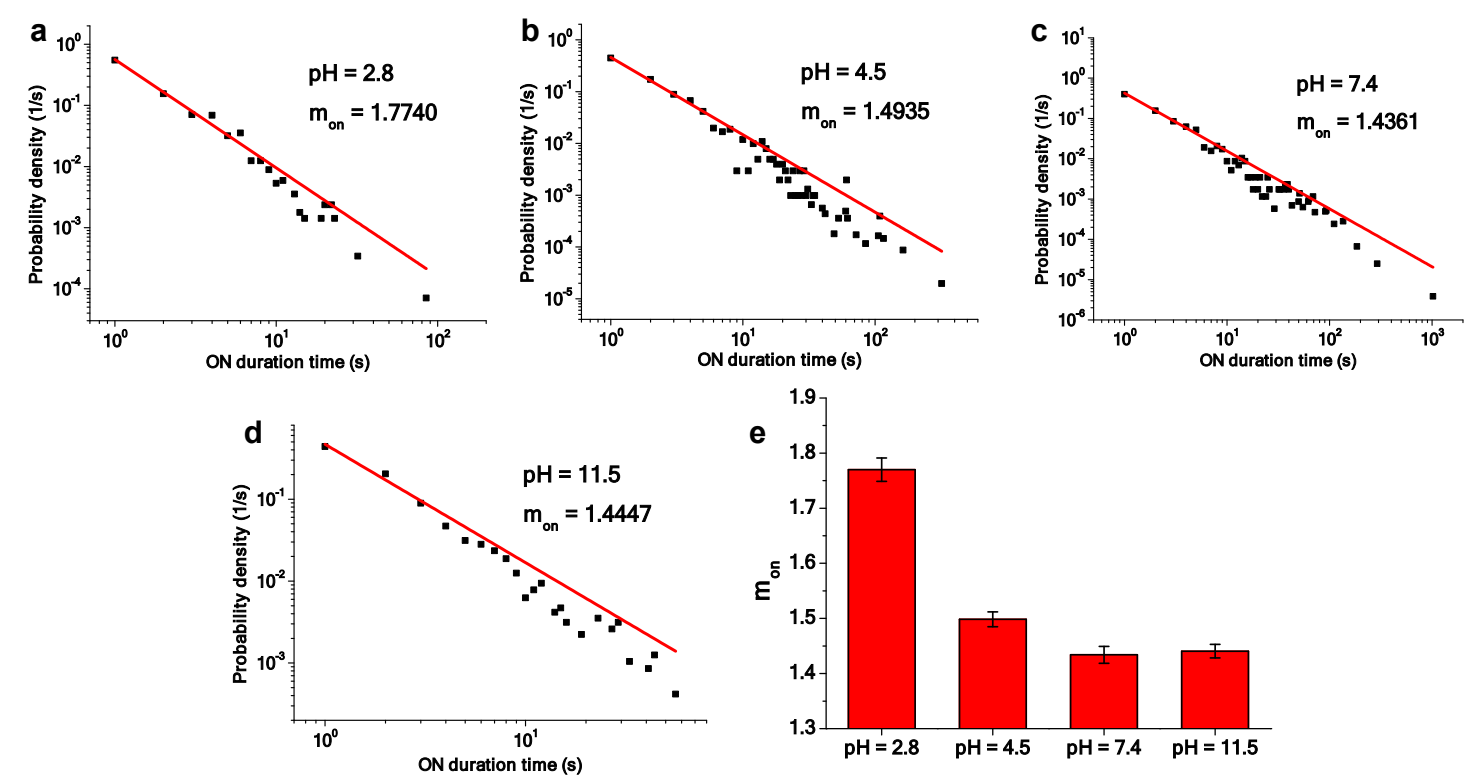

Figure S16. (a-d) Probability density of ON duration time of HCNS in four different $\mathrm{pH}$ environment $(2.8,4.5,7.4$ and 11.5$)$ at $-1.5 \mathrm{~V}$ potential. The dots represent the probability density of different $\mathrm{ON}$ duration times. The lines represent the power-law distribution fitting lines (goodness-of-fit, $\mathrm{R}^{2}$ are 0.997, 0.996, 0.996 and 0.988, respectively). (e) Distribution of power-law coefficient $m_{o n}$ of ECL blinking in four different $\mathrm{pH}$ environment $(2.8,4.5,7.4$ and 11.5) at -1.5 V. Error bars indicate the standard deviation $(\mathrm{n}=5)$. Electrolyte: $100 \mathrm{mM} \mathrm{K}_{2} \mathrm{SO}_{4}$ containing $100 \mathrm{mM}$ 
$\mathrm{K}_{2} \mathrm{~S}_{2} \mathrm{O}_{8}$ coreactant. The $\mathrm{pH}$-value is adjusted by $\mathrm{H}_{2} \mathrm{SO}_{4}$ and $\mathrm{KOH}$ solutions. The exposure time of ECL imaging is 1 s.

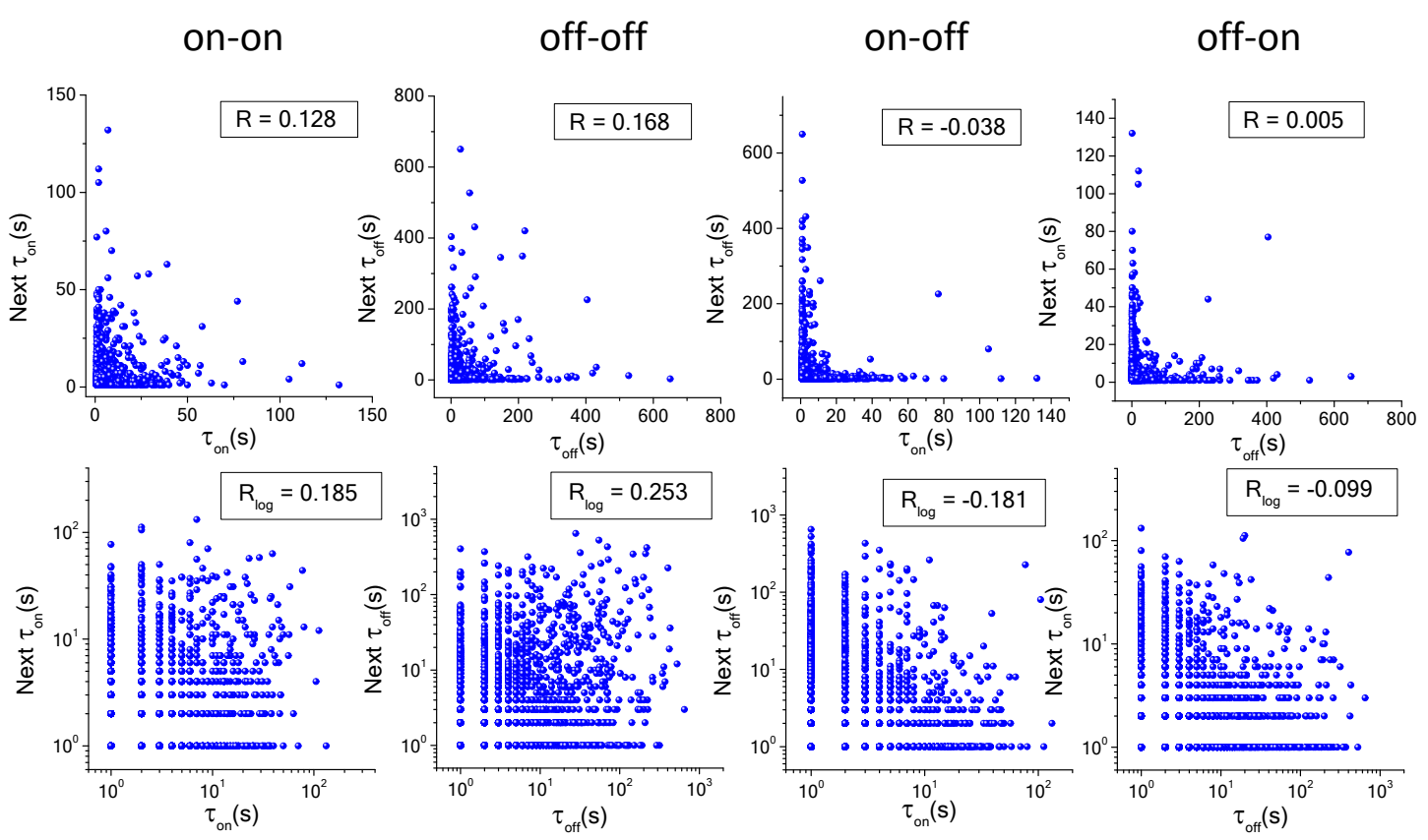

Figure S17. Correlations of adjacent on-on, off-off, on-off, and off-on duration. The linear (Pearson) correlation coefficients $\mathrm{R}$ and $\mathrm{R}_{\log }$ are shown in the corresponding graphs with a linear timescale and a logarithmic timescale, respectively.
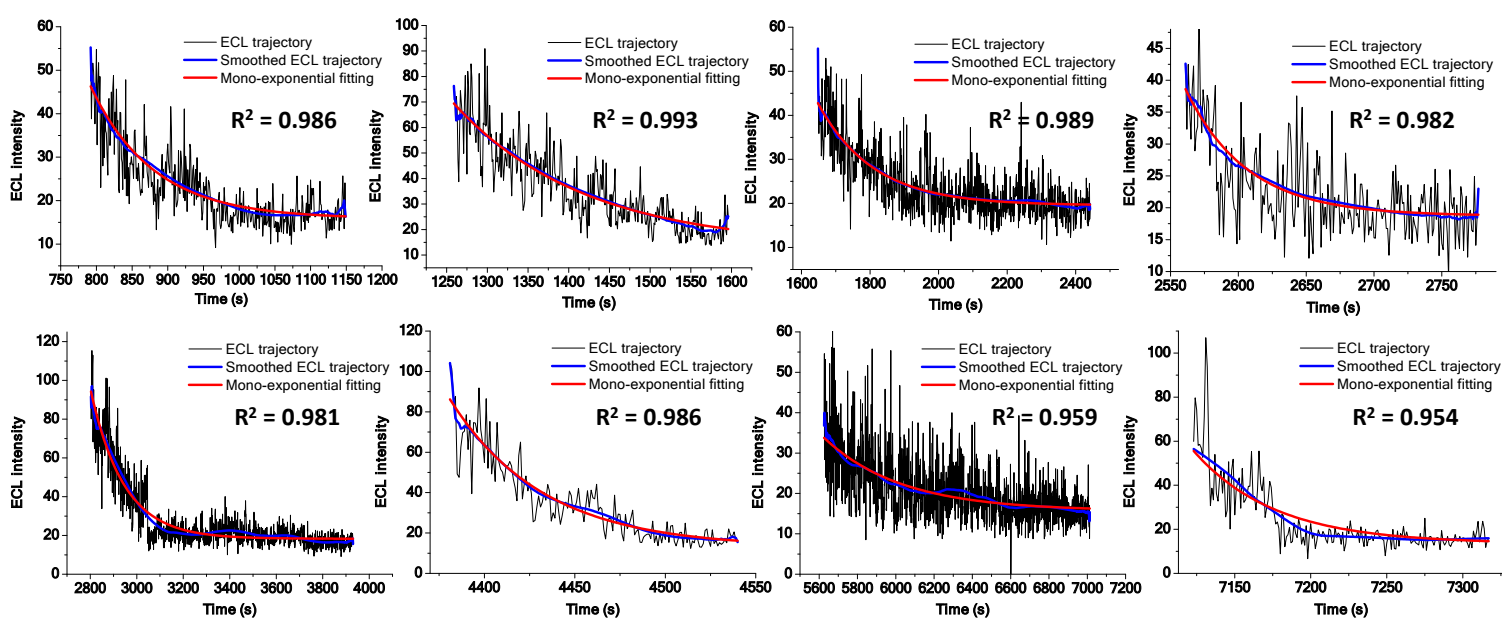

Figure S18. Zoom-in curves of detailed ECL switching events from ON to OFF state at $-1.1 \mathrm{~V}$. The coefficient of determination $\mathrm{R}^{2}$ is used to show the goodness-of-fit of mono-exponential curves. The statistic $\mathrm{R}^{2}$ of monoexponential curves that fit smoothed decay curves are $0.972 \pm 0.020(n=11)$. 

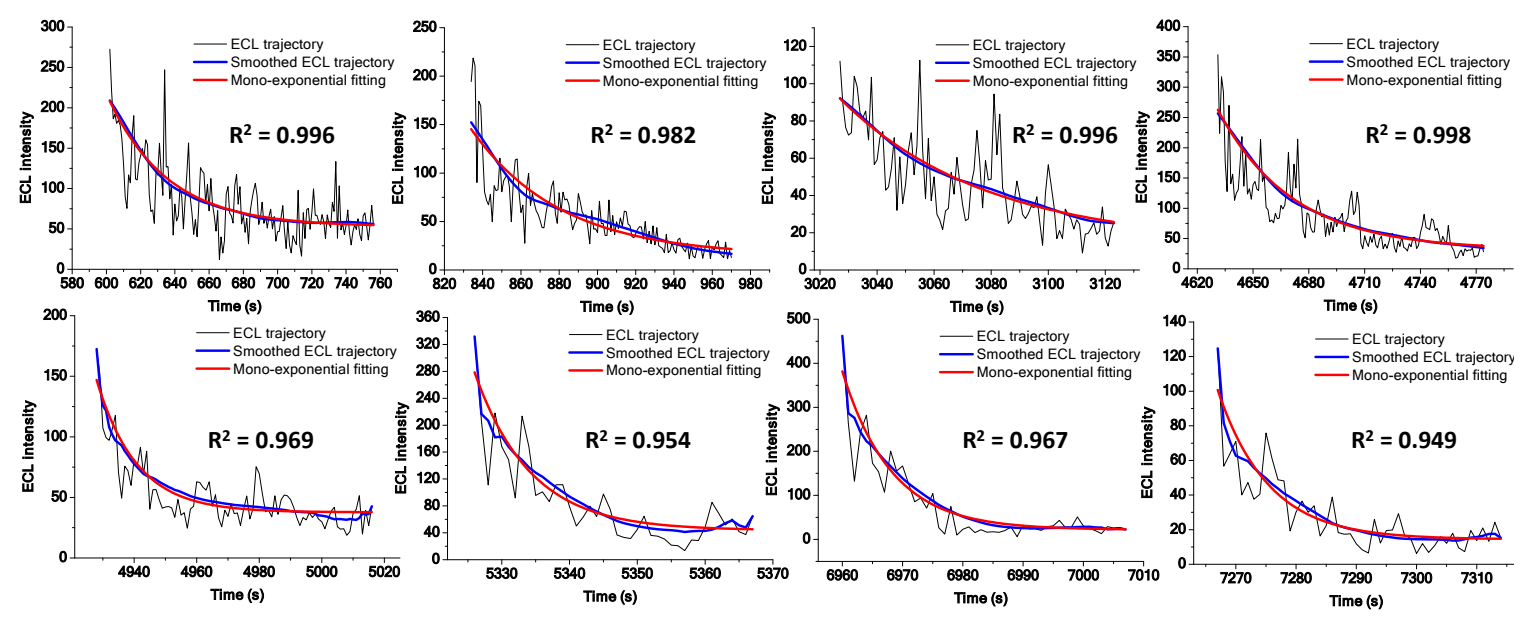

Figure S19. Zoom-in curves of detailed ECL switching events from ON to OFF state at $-1.3 \mathrm{~V}$. The coefficient of determination $\mathrm{R}^{2}$ is used to show the goodness-of-fit of mono-exponential curves. The statistic $\mathrm{R}^{2}$ of monoexponential curves that fit smoothed decay curves are $0.970 \pm 0.020(n=30)$.
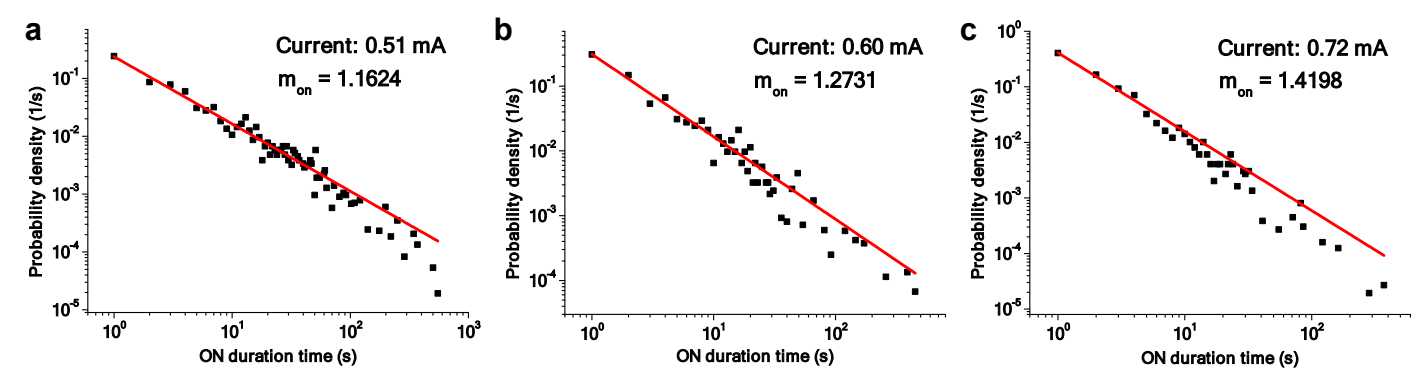

Figure S20. Probability density of $\mathrm{ON}$ duration times with the average currents $(0.51 \mathrm{~mA}, 0.60 \mathrm{~mA}, 0.72 \mathrm{~mA})$ at $1.1 \mathrm{~V}(\mathrm{a}),-1.2 \mathrm{~V}$ (b) and $-1.3 \mathrm{~V}$ (c), respectively. The dots represent the probability density of different ON duration time. The lines represent the power-law distribution fitting lines.

\section{Characterization of AuPd/HCNS, NiS/HCNS and Pt/HCNS.}
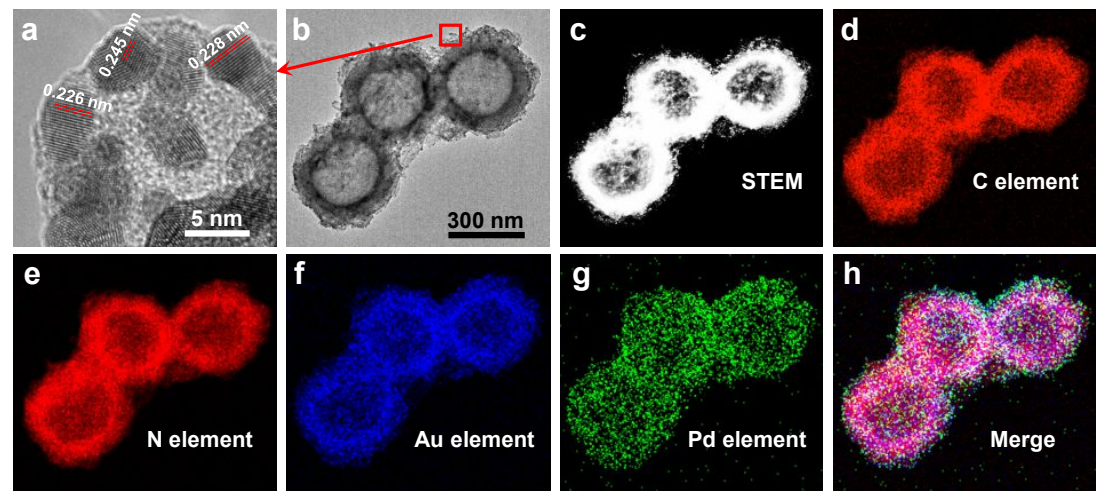

Figure S21. (a,b) HRTEM images of AuPd/HCNSs. The interplanar distance of $0.226 \mathrm{~nm}, 0.228 \mathrm{~nm}$ and $0.245 \mathrm{~nm}$ observed in the HRTEM image (a) belong to AuPd alloy nanoparticles. (c) EDX high-angle annular dark field (HAADF) STEM image of AuPd/HCNSs. (d-h) STEM-EDX elemental maps of selected AuPd/HCNSs. 

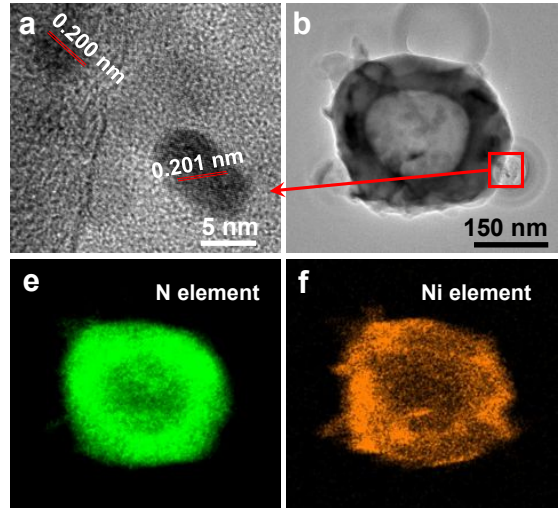
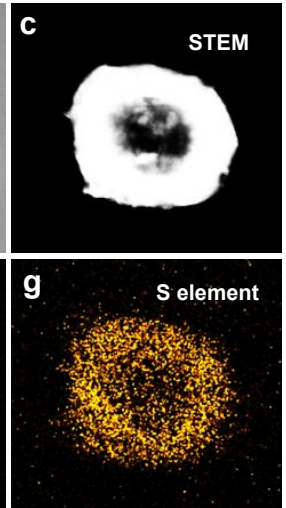

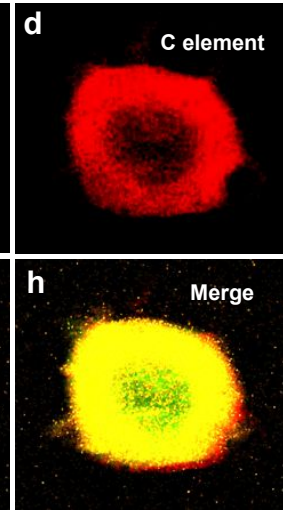

Figure S22. $(a, b)$ HRTEM images of NiS/HCNSs. The interplanar distance of $0.201 \mathrm{~nm}$ observed in the HRTEM image (a) conforms to the (102) crystallographic plane of NiS nanoparticles. (c) EDX high-angle annular dark field (HAADF) STEM image of NiS/HCNSs. (d-h) STEM-EDX elemental maps of selected NiS/HCNSs.
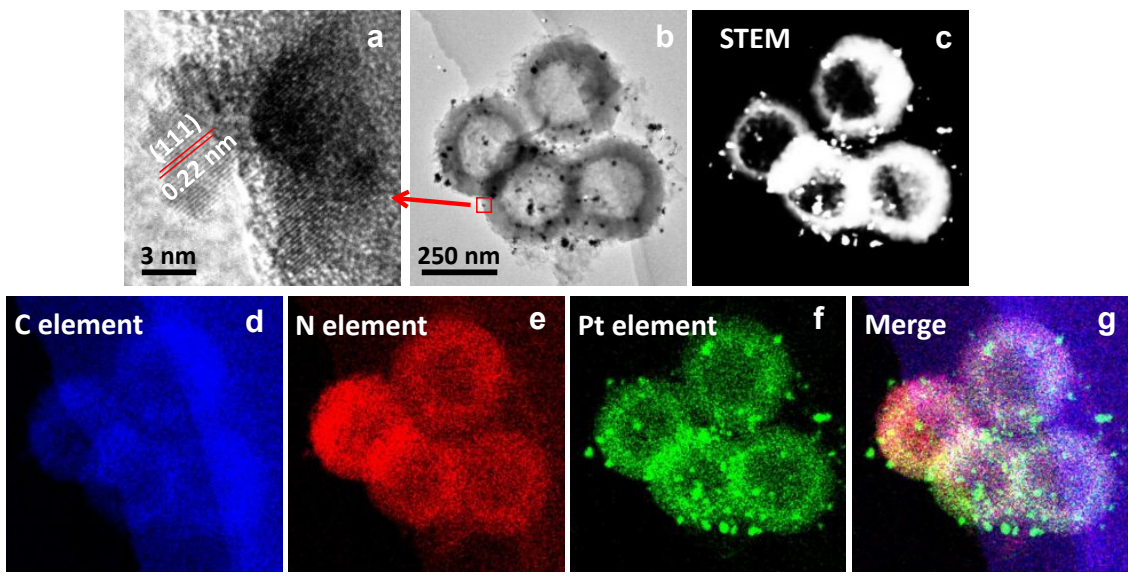

Figure S23. (a,b) HRTEM images of Pt/HCNSs. The interplanar distance of $0.22 \mathrm{~nm}$ observed in the HRTEM image (a) conforms to the (111) crystallographic plane of Pt nanoparticles. (c) EDX high-angle annular dark field (HAADF) STEM image of Pt/HCNSs. (d-g) STEM-EDX elemental maps of selected Pt/HCNSs.

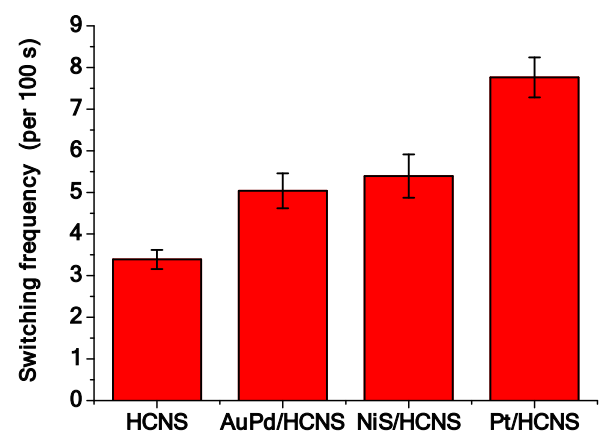

Figure S24. Frequencies of switching events between ON state and OFF state of four nanomaterials. The switching frequencies of HCNSs, AuPd/HCNS, NiS/HCNS and Pt/HCNS are 3.39 $\pm 0.23,5.04 \pm 0.42,5.39 \pm 0.52,7.76 \pm$ 0.48 per $100 \mathrm{~s}(\mathrm{n}=5)$, respectively. 

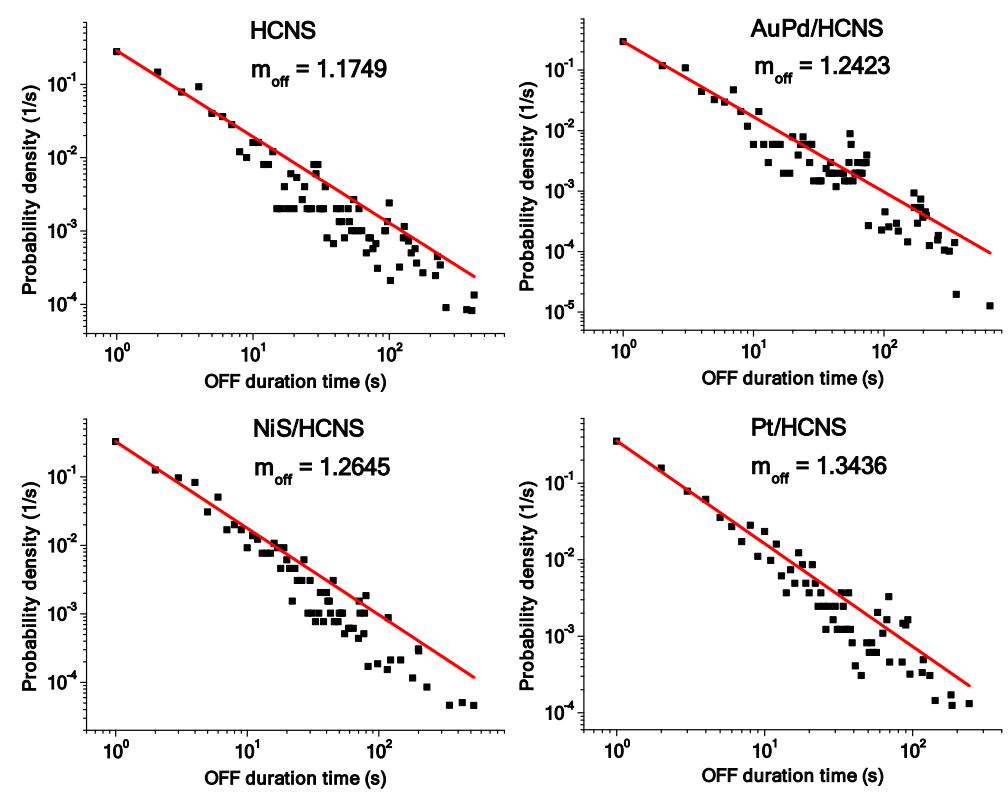

Figure S25. Probability density of OFF duration times of pristine HCNS, AuPd/HCNS, NiS/HCNS and Pt/HCNS. The dots represent the probability density of different OFF duration times. The lines represent the power-law distribution fitting lines. The power-law coefficients $m_{\text {off }}$ of HCNS, AuPd/HCNS, NiS/HCNS and Pt/HCNS are $1.1749,1.2423,1.2645$ and 1.3436 , respectively.

\section{ECL blinking causes the low cathodic ECL efficiency.}

Table S1. ECL maximum ( $\left.\mathrm{ECL}_{\mathrm{max}}\right)$, ECL average $\left(\mathrm{ECL}_{\mathrm{ave}}\right)$, and $\mathrm{ECL}$ efficiency $\left(\mathrm{ECL}_{\mathrm{eff}}\right)$ of eighteen $\mathrm{HCNSs}$ at constant $-1.1 \mathrm{~V},-1.3 \mathrm{~V}$ and $-1.5 \mathrm{~V}$ during $2500 \mathrm{~s} . \mathrm{ECL}_{\text {eff }}$ is defined as the ratio of $E C L_{\text {ave }}$ to $E C L_{\text {max }}: E C L_{e f f}=\frac{E C L_{\text {ave }}}{E C L_{\text {max }}}$

\begin{tabular}{|c|c|c|c|c|c|c|c|c|c|}
\hline & \multicolumn{3}{|c|}{$-1.5 \mathrm{~V}$} & \multicolumn{3}{|c|}{$-1.3 \mathrm{~V}$} & \multicolumn{3}{|c|}{$-1.1 \mathrm{~V}$} \\
\hline & $\mathrm{ECL}_{\text {max }}$ & $\mathrm{ECL}_{\text {ave }}$ & $\mathrm{ECL}_{\text {eff }}$ & $\mathrm{ECL}_{\max }$ & $\mathrm{ECL}_{\text {ave }}$ & $\mathrm{ECL}_{\text {eff }}$ & $\mathrm{ECL}_{\max }$ & $\mathrm{ECL}_{\mathrm{ave}}$ & $\mathrm{ECL}_{\mathrm{eff}}$ \\
\hline HCNS1 & 957.5 & 74.84 & $7.82 \%$ & 608.6 & 68.48 & $11.3 \%$ & 209.7 & 83.14 & $39.6 \%$ \\
\hline HCNS2 & 529.2 & 35.58 & $6.72 \%$ & 568.6 & 65.12 & $11.5 \%$ & 131.7 & 48.18 & $36.6 \%$ \\
\hline HCNS3 & 533.5 & 37.97 & $7.12 \%$ & 735.1 & 78.08 & $10.6 \%$ & 118.8 & 36.97 & $31.1 \%$ \\
\hline HCNS4 & 731.7 & 45.27 & $6.19 \%$ & 341.9 & 53.40 & $15.6 \%$ & 139.6 & 41.40 & $29.7 \%$ \\
\hline HCNS5 & 451.8 & 46.82 & $10.4 \%$ & 425.3 & 45.74 & $10.8 \%$ & 186.4 & 59.36 & $31.9 \%$ \\
\hline HCNS6 & 1276 & 93.22 & $7.31 \%$ & 492.5 & 86.11 & $17.5 \%$ & 246.5 & 51.36 & $20.8 \%$ \\
\hline HCNS7 & 666.4 & 61.04 & $9.16 \%$ & 814.6 & 116.7 & $14.3 \%$ & 287.9 & 74.70 & $25.9 \%$ \\
\hline HCNS8 & 444.9 & 30.87 & $6.94 \%$ & 596.4 & 60.30 & $10.1 \%$ & 161.8 & 61.24 & $37.8 \%$ \\
\hline HCNS9 & 841.5 & 40.59 & $4.82 \%$ & 279.9 & 59.09 & $21.1 \%$ & 665.8 & 60.67 & $9.11 \%$ \\
\hline HCNS10 & 522.5 & 23.58 & $4.51 \%$ & 992.3 & 80.83 & $8.15 \%$ & 694.9 & 121.6 & $17.5 \%$ \\
\hline HCNS11 & 397.7 & 20.95 & $5.27 \%$ & 271.8 & 53.21 & $19.6 \%$ & 160.7 & 39.84 & $24.8 \%$ \\
\hline HCNS12 & 1291 & 54.06 & $4.19 \%$ & 915.3 & 71.69 & $7.83 \%$ & 229.6 & 49.13 & $21.4 \%$ \\
\hline HCNS13 & 591.6 & 27.47 & $4.64 \%$ & 602.6 & 89.70 & $14.9 \%$ & 209.0 & 56.15 & $26.9 \%$ \\
\hline HCNS14 & 1312 & 74.83 & $5.70 \%$ & 447.9 & 42.36 & $9.46 \%$ & 356.4 & 57.53 & $16.1 \%$ \\
\hline HCNS15 & 1014 & 73.49 & $7.25 \%$ & 695.9 & 99.52 & $14.3 \%$ & 199.7 & 74.03 & $37.1 \%$ \\
\hline
\end{tabular}




\begin{tabular}{|c|c|c|c|c|c|c|c|c|c|}
\hline HCNS16 & 519.3 & 29.16 & $5.62 \%$ & 800.6 & 74.00 & $9.24 \%$ & 160.1 & 35.87 & $22.4 \%$ \\
\hline HCNS17 & 1433 & 122.7 & $8.56 \%$ & 747.9 & 76.65 & $10.2 \%$ & 144.1 & 50.59 & $35.1 \%$ \\
\hline HCNS18 & 656.2 & 45.02 & $6.86 \%$ & 544.2 & 62.54 & $11.5 \%$ & 244.5 & 68.44 & $28.0 \%$ \\
\hline Average & 787.2 & 52.08 & $6.62 \%$ & 604.5 & 71.31 & $12.7 \%$ & 252.6 & 59.46 & $27.3 \%$ \\
\hline
\end{tabular}
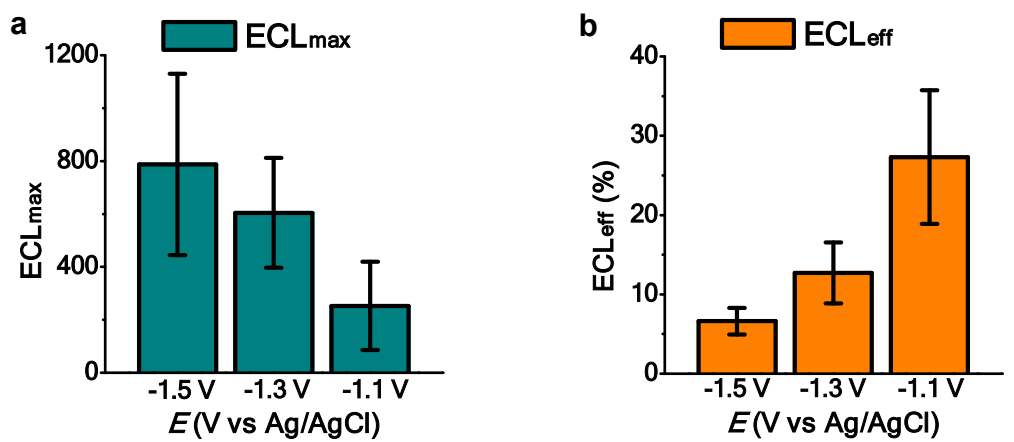

Figure S26. $\mathrm{ECL}_{\max }$ and $\mathrm{ECL}_{\mathrm{eff}}$ statistics of eighteen HCNSs at constant $-1.1 \mathrm{~V},-1.3 \mathrm{~V}$ and $-1.5 \mathrm{~V}$ voltage during $2500 \mathrm{~s}$. Error bars indicate the standard deviation $(\mathrm{n}=18)$.
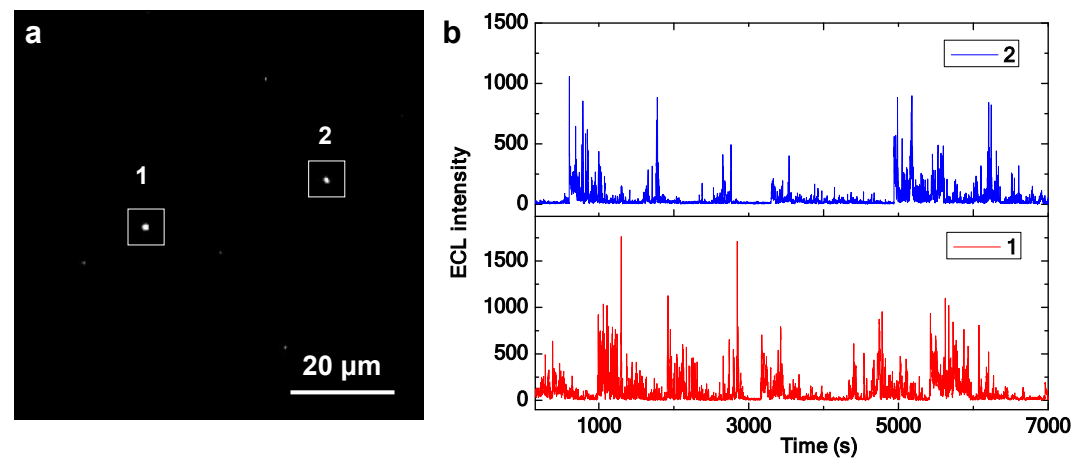

Figure S27. ECL blinking of laminated carbon nitride. (a) Cathodic ECL image of laminated carbon nitride. The ECL image is an accumulation of 10 bright original ECL images to improve the signal to noise ratio. (b) ECL trajectories of two laminated carbon nitride sheets marked in (a) when a constant voltage (-1.5 V) is imposed on the GC electrode. Electrolyte: $100 \mathrm{mM} \mathrm{K}_{2} \mathrm{SO}_{4}$ containing $100 \mathrm{mM} \mathrm{K}_{2} \mathrm{~S}_{2} \mathrm{O}_{8}$ coreactant.
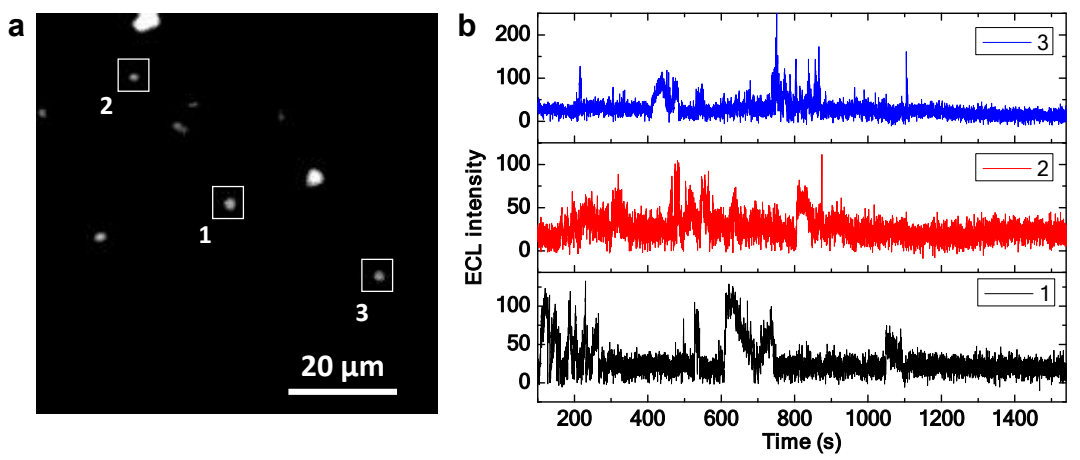
Figure S28. ECL intermittency of CdSe quantum dots aggregates. (a) The cathodic ECL image of CdSe quantum dots aggregates. The ECL image is an accumulation of 20 bright original ECL images to improve the signal to noise ratio. (b) ECL trajectories of three CdSe quantum dots aggregates marked in (a) when a constant voltage (-1.5 V) was imposed on the GC electrode. Electrolyte: $100 \mathrm{mM} \mathrm{K}_{2} \mathrm{SO}_{4}$ containing $100 \mathrm{mM} \mathrm{K}_{2} \mathrm{~S}_{2} \mathrm{O}_{8}$ coreactant.

\section{Preparation and characterization}

Preparation of hollow carbon nitride nanosphere (HCNS) via the hierarchical templating method ${ }^{[1]}$ :

Synthesis of the $\mathrm{SiO}_{2}$ template: Firstly, the nonporous silica core was synthesized according to the Stober method. Briefly, $4.285 \mathrm{~mL}$ of aqueous ammonia (32 wt\%), $74.05 \mathrm{~mL}$ of ethanol and $10 \mathrm{~mL}$ of deionized water were mixed and stirred for $30 \mathrm{~min}$ at $30{ }^{\circ} \mathrm{C}$. Then $5.6 \mathrm{~mL}$ of TEOS was dropwise added to the above mixture under vigorous stirring and subsequently remain stationary for $1 \mathrm{~h}$ to form homodisperse nonporous silica cores. To grow a thin mesoporous silica shell covering the silica core, another batch of TEOS $(4.17 \mathrm{~g})$ and $1.87 \mathrm{~g}$ of $n$ octadecyltrimethoxysilane $\left(\mathrm{C}_{18} \mathrm{TMOS}\right)$ as a porogen were slowly added to the above silica cores solution with vigorous stirring, followed by being left stationary for $3 \mathrm{~h}$ at room temperature. During this period, the precursors TESO and $\mathrm{C}_{18}$ TMOS were cohydrolysed and polymerized on the silica cores with sol-gel process. The as-prepared nanostructured silica materials was centrifuged at $8000 \mathrm{rpm}$ and then dried at $60^{\circ} \mathrm{C}$ in a vacuum oven for $2 \mathrm{~h}$. Finally, the precipitate was calcined at $550^{\circ} \mathrm{C}$ for $6 \mathrm{~h}$ in muffle furnace in air, in order to create a mesoporous structure of silica shell around the dense silica core.

Synthesis of HCNS: $1 \mathrm{~g}$ of the as-prepared $\mathrm{SiO}_{2}$ template was added in $3.9 \mathrm{~mL}$ of cyanamide in a flask and then the flask was connected to a vacuum line with vigorous stirring for $3 \mathrm{~h}$. The vacuum environment facilitates the permeability of cyanamide into the nano-channels of the mesoporous structure. Afterwards, the mixture was sonicated at $60{ }^{\circ} \mathrm{C}$ for $2 \mathrm{~h}$ and stirred overnight at $60{ }^{\circ} \mathrm{C}$, followed by centrifugation and drying at $60{ }^{\circ} \mathrm{C}$ overnight. The white solid products were transferred subsequently to a crucible and gradually heated to $550{ }^{\circ} \mathrm{C}$ for $4 \mathrm{~h}$ with a ramp rate of $4.4{ }^{\circ} \mathrm{C} / \mathrm{min}$ under flowing $\mathrm{N}_{2}$ atmosphere. During this period, the cyanamide underwent thermal polymerization and $\mathrm{g}-\mathrm{C}_{3} \mathrm{~N}_{4} / \mathrm{SiO}_{2}$ hybrids were formed in the end. The obtained pale yellow powder was added to 50 $\mathrm{mL}$ of $4 \mathrm{M} \mathrm{NH}_{4} \mathrm{HF}_{2}$ for $12 \mathrm{~h}$ to etch the silica template. After centrifuged and washed with distilled water and ethanol several times, the obtained HCNSs were collected by drying at $60{ }^{\circ} \mathrm{C}$ in a vacuum oven for $3 \mathrm{~h}$.

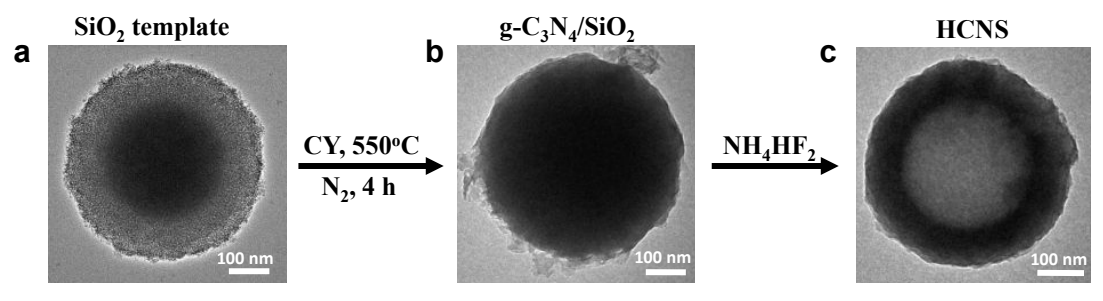

Figure S29. TEM images of synthetic intermediates via the hierarchical templating method. (a) $\mathrm{SiO}_{2}$ template. (b) g- $\mathrm{C}_{3} \mathrm{~N}_{4} / \mathrm{SiO}_{2}$. (c) $\mathrm{HCNS}$.

\section{Loading bimetallic AuPd alloy nanoparticles on HCNS (AuPd/HCNS). ${ }^{[2]}$}

$10 \mathrm{~mL}$ of $\mathrm{HAuCl}_{4}(1 \mathrm{mM})$ and $10 \mathrm{~mL}$ of $\mathrm{PdCl}_{2}(1 \mathrm{mM})$ were mixed and then $728 \mu \mathrm{L}$ of PVA $(1 \mathrm{wt} \%$ aqueous solution) was added into solution. Then $500 \mu \mathrm{L}$ of fresh $\mathrm{NaBH}_{4}(0.1 \mathrm{M})$ was dropwise added to the above mixture under vigorous stirring for $0.5 \mathrm{~h}$ to form homodisperse AuPd alloy nanoparticles. The obtained HCNS powder (10 $\mathrm{mg}$ ) was dispersed and sonicated in $10 \mathrm{~mL}$ of AuPd alloy nanoparticles solution for $0.5 \mathrm{~h}$ to form AuPd/HCNS hybrid. After washing several times with distilled water, the AuPd/HCNS nanomaterials were collected and stored 
in $4{ }^{\circ} \mathrm{C}$.

\section{Loading NiS nanoparticles on HCNS (NiS/HCNS). ${ }^{[3]}$}

The obtained HCNS powder $(6 \mathrm{mg})$ was dispersed in $800 \mu \mathrm{L}$ of distilled water and then $400 \mu \mathrm{L}$ of $\mathrm{Ni}\left(\mathrm{NO}_{3}\right)_{2}$ $(0.02 \mathrm{M})$ was added into the solution. After stirring for $30 \mathrm{~min}$ at room temperature, $240 \mu \mathrm{L}$ of $\mathrm{NaOH}(0.05 \mathrm{M})$ was added into the solution. After stirring for $1 \mathrm{~h}$ at room temperature, $120 \mu \mathrm{L}$ of $\mathrm{Na}_{2} \mathrm{~S}(0.05 \mathrm{M})$ was added into the solution, followed by stirring for another $1 \mathrm{~h}$. After washing several times with distilled water and ethanol, the $\mathrm{NiS} / \mathrm{HCNS}$ nanomaterials were collected and stored in $4{ }^{\circ} \mathrm{C}$.

\section{Loading Pt nanoparticles on HCNS (Pt/HCNS). ${ }^{[1]}$}

The obtained HCNS powder $(5 \mathrm{mg}$ ) was dispersed in $1 \mathrm{~mL}$ of distilled water containing $10 \%$ triethanolamine solution by volume, followed by adding of $100 \mu \mathrm{L}$ of $1 \% \mathrm{H}_{2} \mathrm{PtCl}_{6}$ aqueous solution. To in-situ photodeposit Pt nanoparticles on the HCNS, the mixture was irradiated under a mercury lamp (Nikon Intensilight C-HGFI) equipped with a bandpass filter ( $350 \mathrm{~nm}, 10 \mathrm{~nm}$ FWHM) and a convex lens. After washing several times with distilled water, the $\mathrm{Pt} / \mathrm{HCNS}$ nanomaterials were collected and stored in $4{ }^{\circ} \mathrm{C}$.

\section{Preparation of laminated carbon nitride ${ }^{[4]}$ :}

$30 \mathrm{mg}$ of $\mathrm{g}-\mathrm{C}_{3} \mathrm{~N}_{4}$ bulk powder was exfoliated in $80 \mathrm{~mL}$ of water in a tip ultrasonic processor for $24 \mathrm{~h}$. Then the mixture was centrifuged at $6000 \mathrm{rpm}$ to remove unexfoliated large $\mathrm{g}-\mathrm{C}_{3} \mathrm{~N}_{4}$ particles and aggregates. Afterwards, the supernatant containing laminated carbon nitride was collected and quantified by weighing the power dried from a certain volume of the suspension.

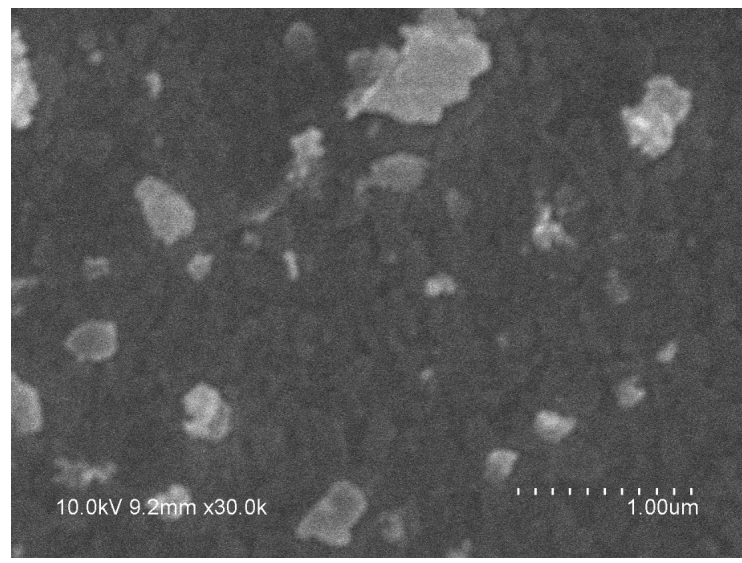

Figure S30. SEM image of laminated carbon nitride.

Preparation of $\mathrm{Ru}(\mathrm{bpy}) 3^{2+}$-doping silica nanoparticles (RuDSNs, $500 \mathrm{~nm}$ diameter) ${ }^{[5]}$ :

APTES $(5 \mu \mathrm{L})$ and Ru-NHS $(5 \mathrm{mg})$ were added to dry DMF $(100 \mu \mathrm{L})$. The reaction mixture was stirred for 1 $\mathrm{h}$ at room temperature. Then, the mixture was added into $2 \mathrm{~mL}$ dry ethanol, followed by the addition of $20 \mu \mathrm{L}$ TEOS. The mixed solution was injected dropwise into $7 \mathrm{~mL}$ dry ethanol solution that contains $420 \mathrm{~nm} \mathrm{SiO}_{2}(20 \mathrm{mg})$ and $100 \mu \mathrm{L} \mathrm{NH}{ }_{4} \mathrm{OH}$. After stirring for $16 \mathrm{~h}$ at $35^{\circ} \mathrm{C}$, the as-prepared RuDSNs were collected by centrifugation at 5000 rpm and washing with water several times. 


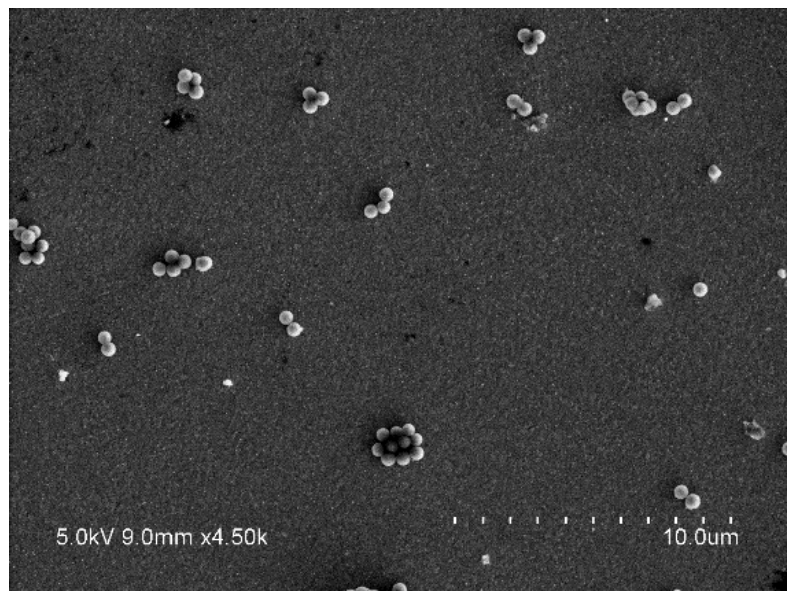

Figure S31. SEM image of $\mathrm{Ru}(\mathrm{bpy}) 3_{3}{ }^{2+}$-doping silica nanoparticle (RuDSN).

\section{Synthesis of CdSe QDs. ${ }^{[6]}$}

In a typical synthesis, $\mathrm{CdCl}_{2}$ aqueous solution $(0.20 \mathrm{M}, 0.80 \mathrm{~mL})$, MPA $(34.6 \mu \mathrm{L})$, and HMP $(72.5 \mathrm{mg})$ were added in $50 \mathrm{~mL}$ of distilled water. Afterwards, the $\mathrm{pH}$ of mixture was adjusted to 9.0 with dropwise adding $1 \mathrm{M}$ $\mathrm{NaOH}$. Then $0.80 \mathrm{~mL}$ of $20.0 \mathrm{mM} \mathrm{Na}_{2} \mathrm{SeO}_{3}$ aqueous solution was added to the solution, followed by being refluxed for $10 \mathrm{~min}$. After cooling to room temperature, $3.67 \mathrm{~mL}$ of $\mathrm{N}_{2} \mathrm{H}_{4} \mathrm{H}_{2} \mathrm{O}$ was added to the above mixture and refluxed for another $10 \mathrm{~h}$. The obtained CdSe QDs was centrifuged at $9500 \mathrm{rpm}$ and washed by isopropyl alcohol several times.
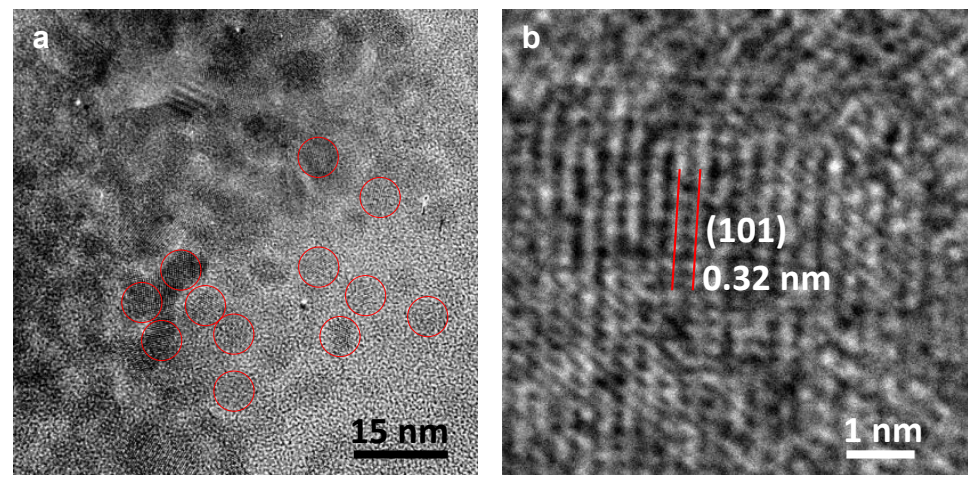

Figure S32. (a,b) HRTEM images of CdSe QDs. The interplanar distance of $0.32 \mathrm{~nm}$ observed in the HRTEM image (b) conforms to the (101) crystallographic plane of CdSe QDs. 

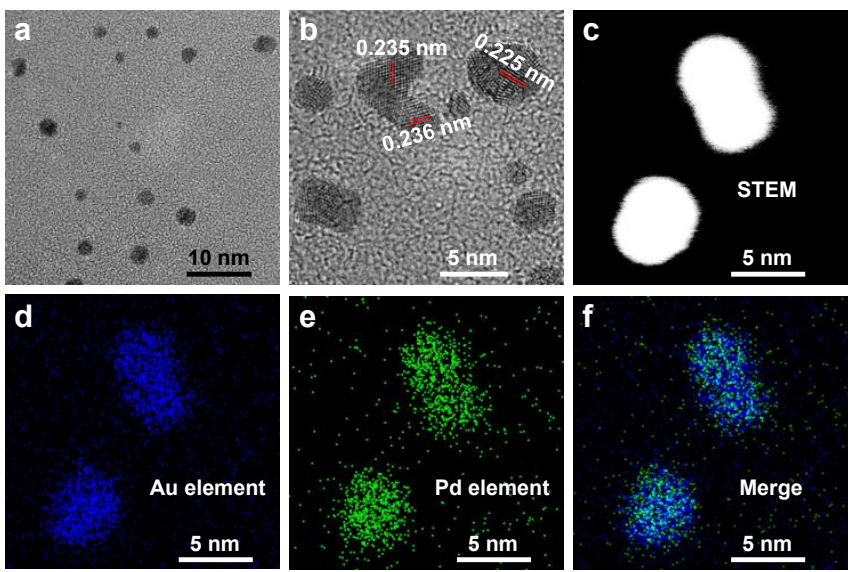

Figure S33. (a,b) HRTEM images of AuPd nanoparticles. The interplanar distance of $0.236 \mathrm{~nm}$ and 0.225 observed in the HRTEM image (b) conforms to the (111) crystallographic plane of Au nanoparticles and Pd nanoparticles, respectively. (c) EDX high-angle annular dark field (HAADF) STEM image of AuPd nanoparticles. (d-f) STEMEDX elemental maps of selected AuPd nanoparticles.

\section{Chemicals and techniques}

Unless otherwise stated, all the other chemicals and reagents used in this study were of analytical grade quality and were used as received without further purification. Ultrapure water with a resistivity of $18.2 \mathrm{M} \Omega \mathrm{cm}$ was produced by using a Milli-Q apparatus (Millipore) and used in the preparation of all solutions. PDMS was prepared by using Sylgard 184, Dow Corning. Tripropylamine (TPrA), bis(2,2'-bipyridine)-4'-methyl-4-carboxybipyridineruthenium N-succinimidyl ester-bis(hexafluorophosphate) (Ru-NHS), mercaptopropionic acid (MPA), and sodium selenite pentahydrate were purchased from Sigma-Aldrich. Tetraethylorthosilicate (TEOS) was purchased from Sinopharm Reagent (Beijing China). Sodium hexametaphosphate (HMP), $n$-octadecyltrimethoxysilane ( $\mathrm{C}_{18} \mathrm{TMOS}$ ), $\mathrm{NH}_{4} \mathrm{HF}_{2}$ were obtained from Macklin Biochemical Co., Ltd. Cadmium chloride was obtained from Jinshanting Chemical Co., Ltd. (Shanghai, China). Cyanamide (CY) was purchased from Alfa Aesar. Graphitic carbon nitride $\left(\mathrm{g}-\mathrm{C}_{3} \mathrm{~N}_{4}\right)$ bulk material was purchased from Nanjing XFNANO Materials Tech Co., Ltd.

Scanning electron microscopy (SEM) micrographs were measured on a Model JSF-7800F scanning electron microscope. High-resolution TEM, HAADF-STEM and STEM-EDX micrographs were measured on a JEM 2800 transmission electron microscope. Electrochemical measurements were performed on a CHI 660D workstation $(\mathrm{CH}$ Instruments Inc., Shanghai, China). The bulk ECL signals were recorded on ECL analyzer (Xi' an Remex Analytical Instrument Co.,Ltd., China) with a three-electrode system. The amount of hydrogen was measured by on-line gas chromatography (Agilent 7890B, $\mathrm{N}_{2}$ carrier). The structures of the HCNSs were confirmed by XRD on a Thermo ARL SCINTAG X'TRA diffractometer using a $\mathrm{Cu}-\mathrm{K} \alpha$ radiation $(\lambda=0.15405 \mathrm{~nm}) . \mathrm{N}_{2}$ adsorption-desorption isotherms were recorded on a Micromeritics ASAP 2020M automated sorption analyzer. The specific surface areas were calculated from the adsorption data in low pressure range using the Brunauer-Emmett-Teller (BET) modal.

Prior to use, GC electrodes ( $3 \mathrm{~mm}$ in diameter) were polished sequentially with 0.3 and $0.05 \mu \mathrm{m}$ alumina slurry on an abrasive cloth, followed by ultrasonic cleaning with ethanol and ultrapure water thoroughly. Then the electrodes were allowed to dry under a $\mathrm{N}_{2}$ flow.

\section{Description of movies}

Movie S1. Electrochemical blinking of single HCNS recorded by ECL microscopy (Work electrode: Glassy carbon 
electrode. Constant potential: $-1.5 \mathrm{~V}$ vs. $\mathrm{Ag} / \mathrm{AgCl}$ reference electrode. Exposure time: $1 \mathrm{~s}$. Electrolyte: $100 \mathrm{mM}$ $\mathrm{K}_{2} \mathrm{SO}_{4}$ containing $100 \mathrm{mM} \mathrm{K}_{2} \mathrm{~S}_{2} \mathrm{O}_{8}$.).

Movie S2. The whole motion process of single HCNS recorded by ECL microscopy. (Work electrode: Glassy carbon electrode. Constant potential: $-1.5 \mathrm{~V}$ vs. $\mathrm{Ag} / \mathrm{AgCl}$ reference electrode. Exposure time: $1 \mathrm{~s}$. Electrolyte: $100 \mathrm{mM}$ $\mathrm{K}_{2} \mathrm{SO}_{4}$ containing $100 \mathrm{mM} \mathrm{K}_{2} \mathrm{~S}_{2} \mathrm{O}_{8}$.).

\section{Reference}

[1] J. Sun, J. Zhang, M. Zhang, M. Antonietti, X. Fu, X. Wang, Nat. Commun. 2012, 3, 1139-1145.

[2] C. Han, L. Wu, L. Ge, Y. Li, Z. Zhao, Carbon 2015, 92, 31-40.

[3] J. Wen, X. Li, H. Li, S. Ma, K. He, Y. Xu, Y. Fang, W. Liu, Q. Gao, Applied Surface Science 2015, 358, 204-212.

[4] L. Chen, X. Zeng, P. Si, Y. Chen, Y. Chi, D. H. Kim, G. Chen, Anal. Chem. 2014, 86, 4188-4195.

[5] C. Ma, W. Wu, L. Li, S. Wu, J. Zhang, Z. Chen, J. J. Zhu, Chem. Sci. 2018, 9, 6167-6175.

[6] S. Liu, X. Zhang, Y. Yu, G. Zou, Anal. Chem. 2014, 86, 2784-2788. 